Original Article

\title{
Bordetella bronchiseptica is a potent and safe adjuvant that enhances the antigen-presenting capability of dendritic cells
}

\author{
You-Jeong Lee', Yong Han', and Hong-Gu Joo ${ }^{1,2, *}$ \\ ${ }^{1}$ College of Veterinary Medicine, ${ }^{2}$ Veterinary Medical Research Institute, Jeju National University, Jeju 63243, Korea
}

\author{
ARTICLE INFO \\ Received July 16, 2019 \\ Revised November 4, 2019 \\ Accepted November 5, 2019 \\ *Correspondence \\ Hong-Gu Joo \\ E-mail: jooh@jejunu.ac.kr
}

\section{Key Words}

Bordetella bronchiseptica

Dendritic cells

Immunostimulation

Safety

Vaccines

\begin{abstract}
We previously demonstrated that Bordetella bronchiseptica (B. bronchiseptica) antigen (Ag) enhances the Mycoplasma hyopneumoniae Ag-specific immune response. The focus of this study was whether acellular bacterin of $B$. bronchiseptica could be used as an adjuvant to increase antigen-presenting capability of dendritic cells (DCs) by increasing the level of activation. The metabolic activity of DCs was increased by B. bronchiseptica, similar to lipopolysaccharide (LPS). Flow cytometry analysis revealed that $B$. bronchiseptica increases the expression of major histocompatibility complex class-2, cluster of differentiation (CD)40, CD54, and CD86 which are closely related to DC-mediated immune responses. $B$. bronchiseptica enhanced the production of cytokines related to adaptive immune responses. Furthermore, the survival rate of B. bronchiseptica-injected groups was $100 \%$ at 15 and $20 \mathrm{mg} / \mathrm{kg}$ doses, whereas that of LPS-injected groups was only $20 \%, 0 \%$ at 15 and $20 \mathrm{mg} / \mathrm{kg}$ doses respectively, and so $B$. bronchiseptica is likely to be safer than LPS. Taken together, these results indicate that $B$. bronchiseptica can be used as an adjuvant to enhance the antigen-presenting capability of DCs. B. bronchiseptica is a candidate for producing vaccines, especially in case of DC-mediating efficacy and safety demands. This study provides researchers and clinicians with valuable information regarding the usage of $B$. bronchiseptica as a safe bacteria-derived immunostimulating agent for developing efficient vaccines.
\end{abstract}

\section{INTRODUCTION}

Vaccines are used to obtain an acquired immunity against certain pathogens [1]. A vaccine contains antigen (Ag) and immunostimulating agent. Vaccine Ags include disease-causing weakened or killed microorganisms, its toxins, or one of its surface proteins [1]. However, some vaccine Ags do not have enough intrinsic capability to stimulate immune system, and thus the vaccines need to contain the immunostimulating agents, called adjuvants [2]. Vaccine adjuvant is added to enhance the efficacy of vaccine at a variety of steps. It extends the presence of Ag at injection sites and helps antigen-presenting cells (APCs) to uptake Ag, and also stimulates immune responses by the increase of cytokine produc- tion and immune-related molecules on the surface of APCs [3]. Vaccine adjuvants have been divided into inorganic substances including alum, cytokines, and bacterial products according to their source types [4].

Bordetella pertussis (B. pertussis) and Mycobacterium bovis ( $M$. bovis) have been used as bacteria-derived immunostimulating agents, partly vaccine adjuvants $[5,6]$. M. bovis is the causative agent of tuberculosis in cattle and Bacillus Calmette-Guerin (BCG) is prepared from an attenuated strain of M. bovis. BCG has been used as a vaccine adjuvant, but its side effects include fibrosis and granuloma, and thus it is not used currently [6-8]. $B$. pertussis is the causative agent of pertussis or whooping cough, and its virulence factors include pertussis toxin, filamentous hae- (i) \$ This is an Open Access article distributed under the terms of the Creative Commons Attribution Non-Commercial License, which permits unrestricted non-commercial use, distribution, and reproduction in any medium, provided the original work is properly cited. Copyright @ Korean J Physiol Pharmacol, pISSN 1226-4512, eISSN 2093-3827
Author contributions: Y.J.L., H.G.J. performed acquisition of data, analysis and/or interpretation of data of in vitro experiments and drafting main parts of the manuscript; Y.H., H.G.J. performed and acquisition/analysis of data of in vivo experiments. Y.J.L., H.G.J. revising the manuscript; H.G.J. performed conception and design of study. 
magglutinin, pertactin, and tracheal cytotoxin. Pertussis toxin is a major toxin of $B$. pertussis and has an adjuvant effect such as antibody response [5,9], but can generate the sensitization leading to anaphylaxis [10].

Bordetella bronchiseptica (B. bronchiseptica) is a similar pathogen to $B$. pertussis and causes respiratory disease [11]. Whereas $B$. pertussis is relatively limited to human, $B$. bronchiseptica affects several animals including dogs, cats, and pigs with different strains [12]. Infection of $B$. bronchiseptica alone does not seriously damage to respiratory system of hosts, but the combined infection with other pathogens can cause significant economic loss $[13,14]$. B. bronchiseptica modulates the phenotypes of macrophages, leading to the inhibition of $\mathrm{CD} 4^{+} \mathrm{T}$ cell proliferation [15].

In a previous study, we demonstrated that $B$. bronchiseptica $\mathrm{Ag}$ can enhance the production of Mycoplasma (M.) hyopneumoniae Ag-specific immunoglobulin G [16]. In this study, we investigated whether formalin-fixed whole-cell and acellular vaccine of $B$. bronchiseptica can improve the Ag-presenting capability of dendritic cells (DCs) and may be a candidate of vaccine adjuvant with immunostimulatory activity.

\section{METHODS}

\section{Animals and materials}

C57BL/6 and Balb/c mice were obtained from OrientBio (Seongnam, Korea) and maintained at our animal facility. 8 to 12week old mice were used for all experiments. Animal experiments in this study were performed in accordance with the Institutional Guideline for Animal Use and Care of Jeju National University (2018-0011, 2018-0045). Lipopolysaccharide (LPS; Sigma-Aldrich, St Louis, MO, USA) was used to as a positive control of immunostimulating agent for DCs. The whole-cell bacterin of $B$. bronchiseptica was prepared by formalin-inactivation and acellular vaccine with sonication (B. bronchiseptica) [16]. The protein amount of $B$. bronchiseptica was measured by Bradford assay.

\section{Preparation of bone marrow-derived DCs}

Bone marrow cells (BMs) were harvested from femur and tibia of C57BL/6 mice, and were used to culture DCs. BMs obtained after lysing red blood cells were counted and cultured into 6-well plates. To culture DCs, the cells were cultured with RPMI 1640 medium containing $5 \%$ fetal bovine serum, $2 \mathrm{mM}$ L-glutamine, $100 \mathrm{U} / \mathrm{ml}$ penicillin-streptomycin, and $10 \mathrm{ng} / \mathrm{ml}$ mouse recombinant granulocyte macrophage-colony stimulating factor (GMCSF; Peprotech, Rocky Hill, NJ, USA). The culture medium was replaced with new medium containing GM-CSF every two days. In order to minimize the interference of lymphocytes and granulocytes in DCs, the floating cells were removed on the 2nd, 4 th day and DC precursor cells attached to the bottom were cultured. After 6 day culture, the floating cells were harvested and used as DCs.

\section{Measurement of DC metabolic activity}

To measure the metabolic activity of DCs, the cells were seeded in 96-well plates and treated with LPS or B. bronchiseptica for 3 days. Then, $5 \mu \mathrm{l} /$ well Cell counting kit-8 (CCK-8; Dojindo Molecular Tech., Kumamoto, Japan) solution was added and incubated at $37^{\circ} \mathrm{C}$ for $4 \mathrm{~h}$. The optical density was measured at $450 \mathrm{~nm}$ with a microplate reader (Multiskan FC; Thermo Fisher Scientific, Waltham, MA, USA).

\section{Measurement of cytokines}

DCs were cultured in 96-well plates to analyze the production of cytokines such as tumor necrosis factor (TNF)-alpha, interleukin (IL)-10, and IL-12. The cells were treated with LPS or B. bronchiseptica for 3 days. The supernatants of treated cells were collected and used for Enzyme-linked immunosorbent assay (ELISA). The amounts of TNF-alpha, IL-10, and IL-12 were determined by CytoSet kits (Thermo Fisher Scientific) according to the instructions. The optical density was measured at $405 \mathrm{~nm}$.

\section{Flow cytometry analysis}

Flow cytometry analysis was performed using surface markerspecific monoclonal antibodies (Abs) to measure the expression of activation and maturation markers of DCs. DCs were cultured in 6-well plates and treated with LPS or B. bronchiseptica for 3 days in the presence of GM-CSF. The treated cells were stained with biotin-labeled anti-MHC class II (I-A ${ }^{\mathrm{b}}$ ), anti-CD40, anti-CD54, or anti-CD86 $\mathrm{Ab}$, and sequentially with streptavidin-fluorescein isothiocyanate (FITC) (all from BD Bioscience, Franklin Lakes, NJ, USA). To measure the antigen uptake capability of DCs, the cells were incubated with FITC-dextran at $250 \mu \mathrm{g} / \mathrm{ml}$ at $37^{\circ} \mathrm{C}$ for $1 \mathrm{~h}$. All stained cells were analyzed by CytoFLEX and CytExpert software (Beckman Coulter, Brea, CA, USA).

\section{Mixed lymphocyte reaction (MLR)}

To evaluate the adjuvanticity of $B$. bronchiseptica, the cultured DCs were treated with LPS, $B$. bronchiseptica for 2 days, and then co-cultured with allogeneic spleen cells. Allogeneic spleen cells were harvested from Balb/c mice. The treated DCs were incubated with $50 \mu \mathrm{g} / \mathrm{ml}$ mitomycin $\mathrm{C}$ for $30 \mathrm{~min}$ before the co-culture setup. The DCs and allogeneic spleen cells were cultured in 96well plates at a concentration of $1 \times 10^{5}$ cells $/ \mathrm{ml}$ and $2 \times 10^{6}$ cells/ $\mathrm{ml}$, respectively. As a control, only allogeneic spleen cells were cultured. After 5 days, $10 \mu \mathrm{l} /$ well of CCK- 8 solution was added and incubated at $37^{\circ} \mathrm{C}$ for $4 \mathrm{~h}$. The optical density was measured 
at $450 \mathrm{~nm}$ with a microplate reader.

\section{Injection of LPS or B. bronchiseptica into mice}

7-week old Balb/c mice were divided into 6 groups. B. bronchiseptica or LPS was injected at 10, 15 and $20 \mathrm{mg} / \mathrm{kg}$ doses respectively, by a single intraperitoneal injection. The mice were monitored for one week to check mortality rate and weight change.

\section{Statistical analysis}

The data of CCK- 8 assay and ELISA are presented as mean \pm standard deviation and were analyzed with one-way analysis of variance, Tukey's multiple comparison test (InStat; GraphPad Software, San Diego, CA, USA). A p-value of $<0.05$ was considered as significant. ${ }^{* *},{ }^{* *}$ indicate $\mathrm{p}<0.01,0.001$ respectively, compared to control.

\section{RESULTS}

\section{Effect of B. bronchiseptica on the metabolic activity of DCs}

In order to determine whether $B$. bronchiseptica affects the metabolic activity of DCs, we performed CCK-8 assays. DCs were treated with $B$. bronchiseptica or LPS for 3 days at a range of concentration, 0 to $300 \mathrm{ng} / \mathrm{ml}$, and the metabolic activity of DCs was measured. B. bronchiseptica at 33.3 and $100 \mathrm{ng} / \mathrm{ml}$ significantly increased the metabolic activity of DCs compared to control (0 $\mathrm{ng} / \mathrm{ml}$ ) (Fig. 1). The highest increase of metabolic activity was appeared at $100 \mathrm{ng} / \mathrm{ml}$, an optimal concentration used for DC activation in this study.

\section{B. bronchiseptica increases the expression of immune response-related surface markers of DCs}

To determine whether $B$. bronchiseptica induces the activation and maturation of DCs, we measured the expression level of surface markers on DCs. The cells were treated with $0.1 \mu \mathrm{g} / \mathrm{ml}$ of $B$. bronchiseptica or LPS for 2 days and stained with surface marker-specific Abs. B. bronchiseptica strongly increased the surface markers expression on DCs, which increase the MHC class 2 molecule by $67 \%$ compared to control, and also other 3 molecules more than $150 \%$ (Fig. 2). This data showed that LPS also had effects on the activation of DCs, but $B$. bronchiseptica increased the expression level of the 4 surface markers higher than LPS.

\section{B. bronchiseptica decreases the antigen uptake capability of DCs}

Antigen uptake assay was performed to confirm whether $B$. bronchiseptica induces the maturation of DCs. The cells were incubated with FITC-dextran at $37^{\circ} \mathrm{C}$ for $1 \mathrm{~h}$. Both LPS and $B$. bronchiseptica showed lower levels of the uptake of FITC-dextran compared to that of control (Fig. 3). The uptake of FITC-dextran in DCs at $4^{\circ} \mathrm{C}$ was used as negative control.

\section{B. bronchiseptica enhances the cytokine production of DCs}

To determine the effect of $B$. bronchiseptica as an adjuvant for vaccines, we measured the production of TNF-alpha, IL-10, and IL-12 in DCs treated with B. bronchiseptica or LPS. ELISA results demonstrated that both $B$. bronchiseptica and LPS increased the production of TNF-alpha, IL-10, and IL-12 (Fig. 4). In the case of TNF-alpha, $B$. bronchiseptica produced less TNF-alpha than LPS at $0.02 \mu \mathrm{g} / \mathrm{ml}$, but produced more at 0.1 and $0.5 \mu \mathrm{g} / \mathrm{ml}$. Interestingly, IL-10 production was significantly elevated in $B$. bronchiseptica-treated DCs compared to LPS-treated DCs at $0.5 \mu \mathrm{g} / \mathrm{ml}$. B. bronchiseptica enhanced the IL-12 production of DCs higher than LPS in all concentrations.

\section{Effect of B. bronchiseptica on the antigen-presenting capability of DCs}

To evaluate whether $B$. bronchiseptica can increase the antigenpresenting capability of DCs, we co-cultured the treated DCs and allogeneic spleen cells. DCs were pre-treated with $B$. bronchiseptica or LPS at $0.1 \mu \mathrm{g} / \mathrm{ml}$ for 2 days, and then co-cultured at a ratio of 1:20 with allogeneic spleen cells harvested from $\mathrm{Balb} / \mathrm{c}$ mice. Mitomycin C was used to limit the metabolic activity of DCs itself and inhibit further maturation during the co-cultures. Both $B$. bronchiseptica- or LPS-treated DCs significantly increased the metabolic activity and proliferation of allogeneic spleen cells compared to control DCs (Fig. 5). This result demonstrated that $B$.

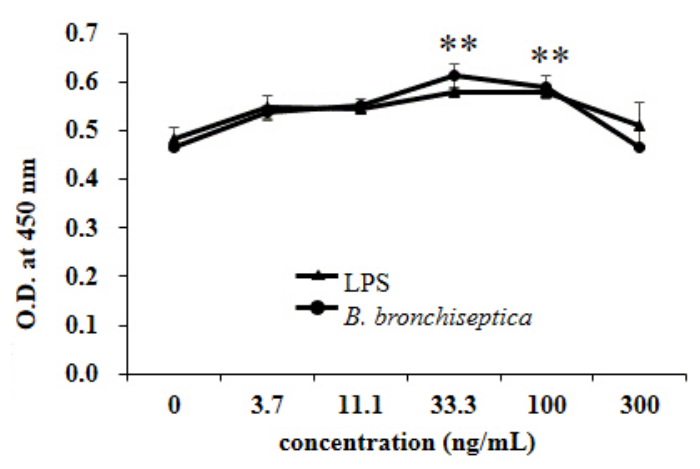

Fig. 1. The effect of Bordetella bronchiseptica (B. bronchiseptica) on the metabolic activity of dendritic cells (DCs). DCs were cultured in 96-well plates at a concentration of $1.5 \times 10^{5}$ cells $/ \mathrm{ml}$ with 0 to 300 $\mathrm{ng} / \mathrm{ml}$ B. bronchiseptica or lipopolysaccharide (LPS) for 3 days. The treated DCs were incubated with $5 \mu \mathrm{l} /$ well CCK-8 solution at $37^{\circ} \mathrm{C}$ for 4 h. The optical density (O.D.) was measured at $450 \mathrm{~nm}$ by using a microplate reader. ${ }^{* *}$ Indicates $p<0.01$. 

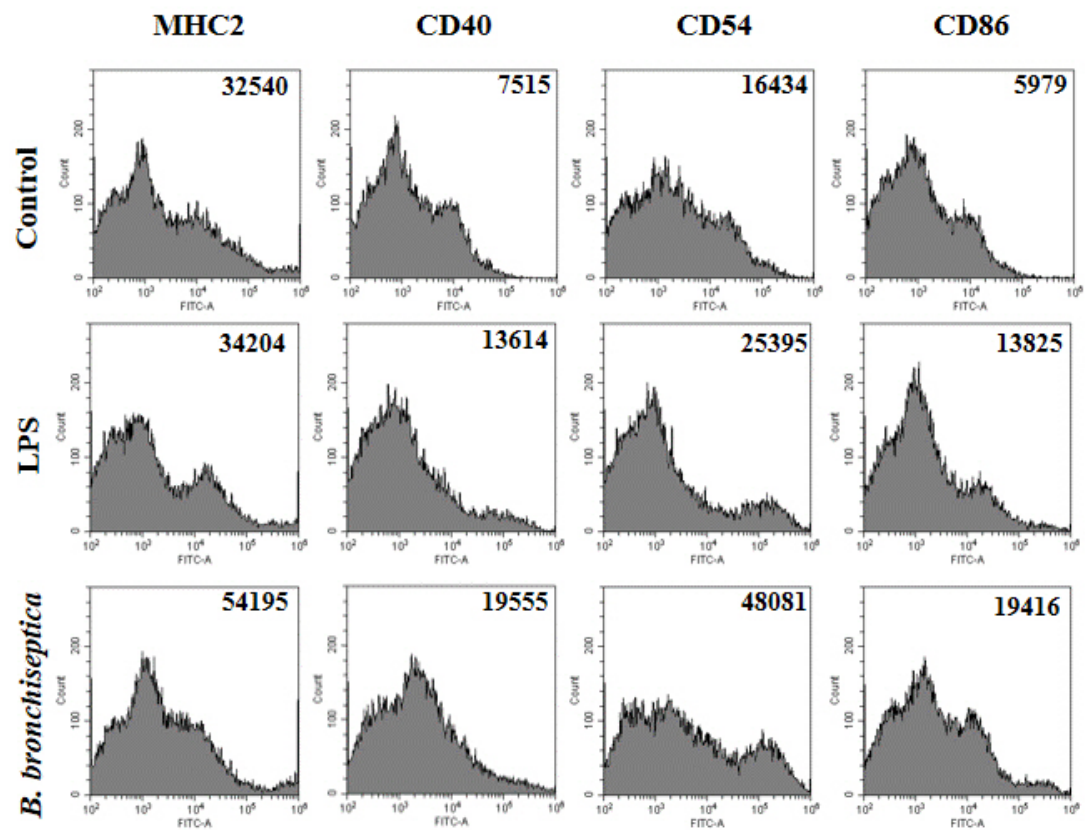

Fig. 2. Effect of Bordetella bronchiseptica (B. bronchiseptica) on the expression of immune response-related surface markers of dendritic cells (DCs). DCs were cultured in 6-well plates at a concentration of $2.5 \times 10^{5}$ cells $/ \mathrm{ml}$ and treated with $0.1 \mu \mathrm{g} / \mathrm{ml}$ B. bronchiseptica or lipopolysaccharide (LPS) for 2 days. After the treatment, DCs were stained as described in "Methods". Numbers of histograms represent the geometric mean fluorescence intensity.

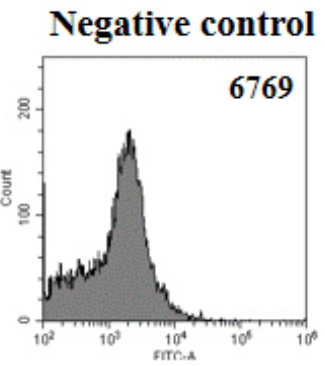

LPS

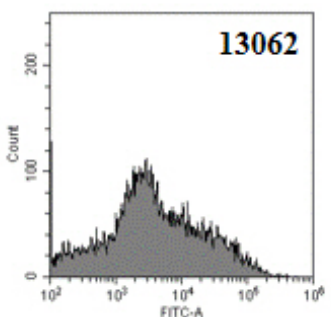

Dextran-FITC

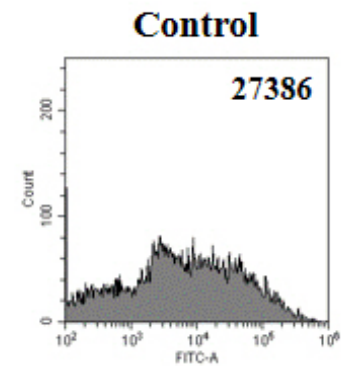

B. bronchiseptica

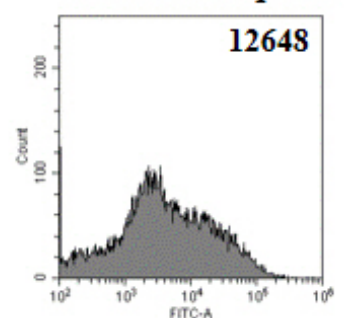

Fig. 3. Bordetella bronchiseptica (B. bronchiseptica) increases the level of dendritic cell (DC) maturation. DCs were cultured in 6-well plates at a concentration of $5.5 \times 10^{5}$ cells/ml and treated with $0.1 \mu \mathrm{g} /$ $\mathrm{ml}$ of $B$. bronchiseptica or lipopolysaccharide (LPS) for 2 days. After treatment, the DCs were incubated with fluorescein isothiocyanate (FITC)-dextran as described in "Methods". Numbers of histograms represent the geometric mean fluorescence intensity. Negative control DCs was treated at $4^{\circ} \mathrm{C}$, and others were treated at $37^{\circ} \mathrm{C}$ for $1 \mathrm{~h}$. bronchiseptica can increase the antigen-presenting capability of DCs.

\section{B. bronchiseptica is safe in vivo}

To investigate if $B$. bronchiseptica is safe in vivo, Balb/c mice were injected with 10,15 , and $20 \mathrm{mg} / \mathrm{kg}$ of $B$. bronchiseptica or LPS. After 7 days, the survival rate and weight change were examined. All mice were survived in both $B$. bronchiseptica- or LPS-injected groups at $10 \mathrm{mg} / \mathrm{kg}$ dose. B. bronchiseptica-injected groups showed $100 \%$ survival rate at 15 and $20 \mathrm{mg} / \mathrm{kg}$ dose, whereas LPS-injected groups showed only $20 \%, 0 \%$ survival rate at 15 and $20 \mathrm{mg} / \mathrm{kg}$ dose respectively (Fig. 6). Interestingly, the body weight change of $B$. bronchiseptica-injected mice was less than $3 \%$ decrease. This result indicates that $B$. bronchiseptica is safer in vivo than LPS.

\section{DISCUSSION}

Adjuvants increase the effects of vaccines by enhancing antigen-specific immune responses, such as maximal $\mathrm{T}$ celldependent immune responses [3]. This effect of vaccine adjuvant can be achieved through several critical events including the activation of antigen-presenting cells such as DCs [17]. After uptaking antigens, DCs migrate to lymph nodes and present antigens to lymphocytes for initiating adaptive immune responses [18]. In previous studies, we demonstrated that $B$. bronchiseptica antigens 

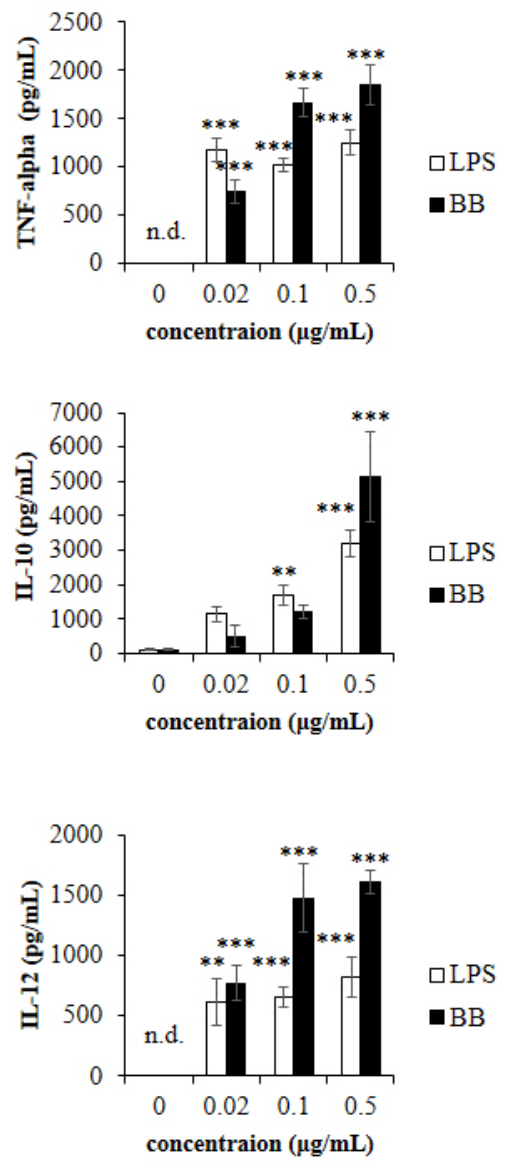

Fig. 4. The effect of Bordetella bronchiseptica (B. bronchiseptica) on the production of immune-related cytokines. Dendritic cells (DCs) were cultured in 96-well plates at a concentration of $5 \times 10^{5}$ cells $/ \mathrm{ml}$ and treated with 0 to $0.5 \mu \mathrm{g} / \mathrm{ml} \mathrm{B}$. bronchiseptica (BB) or lipopolysaccharide (LPS) for 3 days. The supernatant of cells treated with BB or LPS was collected and used for ELISA. The optical density was measured at $405 \mathrm{~nm}$ by using microplate reader. TNF, tumor necrosis factor; IL, interleukin. ${ }^{* *}$, ${ }^{* * *}$ Indicate $p<0.01, p<0.001$, respectively.

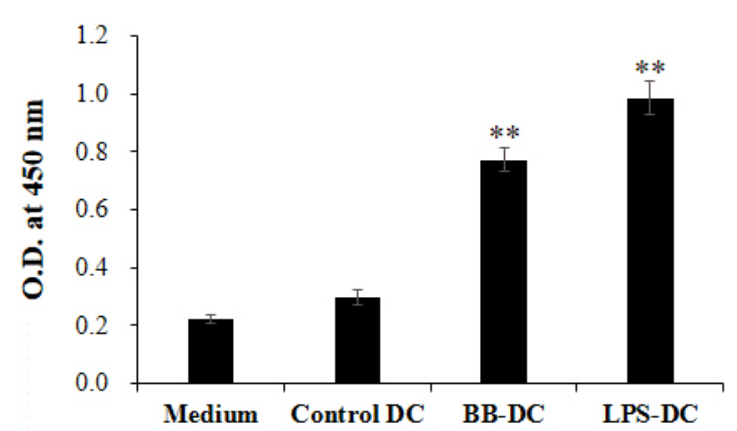

Fig. 5. Effect of Bordetella bronchiseptica (B. bronchiseptica) on the antigen-presenting capability of dendritic cells (DCs). DCs were treated with $0.1 \mu \mathrm{g} / \mathrm{ml}$ of LPS or B. bronchiseptica (BB) for 2 days. The treated DCs were incubated with $50 \mu \mathrm{g} / \mathrm{ml}$ mitomycin $C$ for $30 \mathrm{~min}$ before the co-culture setup. Allogeneic spleen cells were harvested from Balb/c mice. DCs and allogeneic spleen cells were co-cultured in 96well plates at a $1 \times 10^{5}$ cells $/ \mathrm{ml}$ and $2 \times 10^{6}$ cells $/ \mathrm{ml}$ respectively. After 5 days, CCK-8 assay was performed as described in 'Methods'. O.D., optical density. ${ }^{* *}$ Indicates $p<0.01$

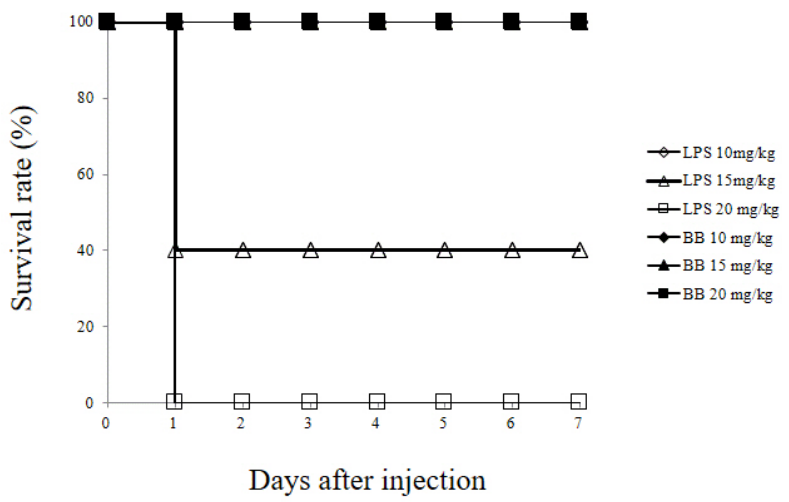

Fig. 6. Bordetella bronchiseptica (B. bronchiseptica) is safe in vivo. 7-week old Balb/c mice were intraperitoneally injected with $B$. bronchiseptica (BB) or lipopolysaccharide (LPS) at 10, 15, and $20 \mathrm{mg} / \mathrm{kg}$ dose respectively. The survival rate of the mice groups was determined at 7 day.

increase the production of M. hyopneumoniae-specific IgG [16]. In this study, we investigated whether $B$. bronchiseptica can be used as an adjuvant to increase the antigen-presenting capability of DCs in several aspects.

This study demonstrated that $B$. bronchiseptica up-regulated the functions of DCs including metabolic activity. CCK-8 assay showed that $B$. bronchiseptica increased the metabolic activity of DCs at a level similar to that of LPS. Flow cytometry analysis showed that $B$. bronchiseptica stimulated the expression of MHC class II, an essential antigen-presenting molecule, on the surface of DCs and increased the expression of DC activation markers such as CD40, CD54, and CD86. In addition, the antigen uptake of $B$. bronchiseptica-treated DCs was consistently low. The matured DCs have much lower antigen uptake capability than immature DCs. Our data collectively indicates that $B$. bronchiseptica can increase the degree of activation and maturation of DC, similar to LPS.

TNF-alpha, IL-10 and IL-12 production were measured to determine how much $B$. bronchiseptica can activate DCs. ELISA showed that $B$. bronchiseptica strongly increased the production of TNF-alpha, IL-10, IL-12. Our data indicate that B. bronchiseptica is effective as an adjuvant to activate DCs. Interestingly IL-10 production was significantly elevated in the DCs treated with $B$. bronchiseptica compared to LPS at $0.5 \mu \mathrm{g} / \mathrm{ml}$. IL-10 is a representative anti-inflammatory cytokine and its production is closely connected to the production of inflammatory cytokine including TNF-alpha $[19,20]$. This result suggests that $B$. bronchiseptica may have a unique regulatory role in the production of inflammatory and anti-inflammatory cytokines.

The immune enhancing effect of $B$. bronchiseptica is similar to or higher than that of LPS. B. bronchiseptica does have less cytotoxicity than LPS on DCs. B. bronchiseptica is a Gram-negative bacterium containing LPS and is likely to act as an immune stimulating factor. It is also suggested that the LPS of $B$. bronchiseptica may have a different structure or content compared to 
that of E. coli. In the future, we will study which components of B. bronchiseptica including LPS have the immunostimulatory effect.

In addition, we measured the effect of $B$. bronchiseptica on the antigen-presenting capability of DCs. MLRs measured by CCK-8 assay showed that $B$. bronchiseptica- and LPS-treated DCs increased the metabolic activity and proliferation of allogeneic lymphocytes. Our data indicate that $B$. bronchiseptica can enhance the antigen-presenting capability of DCs.

To confirm if $B$. bronchiseptica is safe in vivo, mice were injected with the same dose of $B$. bronchiseptica or LPS. Interestingly, B. bronchiseptica-injected mice did not die at all doses $(10,15,20$ $\mathrm{mg} / \mathrm{kg}$ ), whereas LPS-injected mice seriously did at 15 and $20 \mathrm{mg} /$ $\mathrm{kg}$ (survival rate only 20\%,0\% respectively). This result suggests that $B$. bronchiseptica is much safer in vivo than LPS.

Taken together, our data demonstrate that $B$. bronchiseptica enhances the antigen-presenting capability of DCs and has immunostimulatory activity as an adjuvant. In addition, the safety of $B$. bronchiseptica is better than LPS which is already used as a kind of adjuvant (Supplementary Fig. 1). This study shows the availability of $B$. bronchiseptica as a vaccine adjuvant based on its activity on DCs.

\section{ACKNOWLEDGEMENTS}

This work was supported by Korea Institute of Planning and Evaluation for Technology in Food, Agriculture, Forest and Fisheries (IPET) through Agri-Bio industry Technology Development Program, funded by Ministry of Agriculture, Food and Rural Affairs (MAFRA) (Grant number 111065-3, 109014-03-1-CG000).

\section{CONFLICTS OF INTEREST}

The authors declare no conflicts of interest.

\section{SUPPLEMENTARY MATERIALS}

Supplementary data including one figure can be found with this article online at http://pdf.medrang.co.kr/paper/pdf/Kjpp/ Kjpp2020-24-01-06-s001.pdf.

\section{REFERENCES}

1. Karch CP, Burkhard P. Vaccine technologies: from whole organisms to rationally designed protein assemblies. Biochem Pharmacol. 2016;120:1-14.

2. Marciani DJ. Vaccine adjuvants: role and mechanisms of action in vaccine immunogenicity. Drug Discov Today. 2003;8:934-943.
3. Del Giudice G, Rappuoli R, Didierlaurent AM. Correlates of adjuvanticity: a review on adjuvants in licensed vaccines. Semin Immunol. 2018;39:14-21.

4. Coffman RL, Sher A, Seder RA. Vaccine adjuvants: putting innate immunity to work. Immunity. 2010;33:492-503.

5. Dunne A, Mielke LA, Allen AC, Sutton CE, Higgs R, Cunningham CC, Higgins SC, Mills KH. A novel TLR2 agonist from Bordetella pertussis is a potent adjuvant that promotes protective immunity with an acellular pertussis vaccine. Mucosal Immunol. 2015;8:607617.

6. Wangoo A, Brown IN, Marshall BG, Cook HT, Young DB, Shaw RJ. Bacille Calmette-Guérin (BCG)-associated inflammation and fibrosis: modulation by recombinant BCG expressing interferon-gamma (IFN-gamma). Clin Exp Immunol. 2000;119:92-98.

7. Filardi MJ, Codish SD, Civerchia L, Howard RK, McKneally MF. Toxicity of intrapleural Bacillus Calmette-Guérin treatment in animals. Cancer Res. 1979;39:3673-3676.

8. Gordon S, Keshav S, Stein M. BCG-induced granuloma formation in murine tissues. Immunobiology. 1994;191:369-377.

9. Fedele G, Celestino I, Spensieri F, Frasca L, Nasso M, Watanabe M, Remoli ME, Coccia EM, Altieri F, Ausiello CM. Lipooligosaccharide from Bordetella pertussis induces mature human monocyte-derived dendritic cells and drives a Th2 biased response. Microbes Infect. 2007;9:855-863.

10. Samore MH, Siber GR. Pertussis toxin enhanced IgG1 and IgE responses to primary tetanus immunization are mediated by interleukin-4 and persist during secondary responses to tetanus alone. Vaccine. 1996;14:290-297.

11. Zhao Z, Wang C, Xue Y, Tang X, Wu B, Cheng X, He Q, Chen H. The occurrence of Bordetella bronchiseptica in pigs with clinical respiratory disease. Vet J. 2011 188:337-340.

12. Goodnow RA. Biology of Bordetella bronchiseptica. Microbiol Rev. 1980;44:722-738.

13. Chanter N, Magyar T, Rutter JM. Interactions between Bordetella bronchiseptica and toxigenic Pasteurella multocida in atrophic rhinitis of pigs. Res Vet Sci. 1989;47:48-53.

14. Horiguchi Y. Swine atrophic rhinitis caused by pasteurella multocida toxin and bordetella dermonecrotic toxin. Curr Top Microbiol Immunol. 2012;361:113-129.

15. Siciliano NA, Skinner JA, Yuk MH. Bordetella bronchiseptica modulates macrophage phenotype leading to the inhibition of CD4+ T cell proliferation and the initiation of a Th17 immune response. $J$ Immunol. 2006177:7131-7138.

16. Yim SH, Hahn TW, Joo HG. Bordetella bronchiseptica antigen enhances the production of Mycoplasma hyopneumoniae antigenspecific immunoglobulin G in mice. J Vet Sci. 2017;18:327-332.

17. Gallucci S, Lolkema M, Matzinger P. Natural adjuvants: endogenous activators of dendritic cells. Nat Med. 1999;5:1249-1255.

18. Théry C, Amigorena S. The cell biology of antigen presentation in dendritic cells. Curr Opin Immunol. 2001;13:45-51.

19. de Waal Malefyt R, Abrams J, Bennett B, Figdor CG, de Vries JE. Interleukin 10(IL-10) inhibits cytokine synthesis by human monocytes: an autoregulatory role of IL-10 produced by monocytes. J Exp Med. 1991;174:1209-1220.

20. Fiorentino DF, Zlotnik A, Mosmann TR, Howard M, O'Garra A. IL-10 inhibits cytokine production by activated macrophages. J Immunol. 1991;147:3815-3822. 